\title{
Pathophysiology Underpinning Gestational Diabetes Mellitus and the Role of Biomarkers for its Prediction
}

\author{
Authors: \\ *Natassia Rodrigo, ${ }^{1,2,3}$ Sarah J. Glastras ${ }^{1,2,3}$ \\ 1. Department of Diabetes, Endocrinology \& Metabolism, Royal North Shore Hospital, \\ St Leonards, Sydney, Australia \\ 2. The Kolling Institute of Medical Research, St Leonards, Sydney, Australia \\ 3. Faculty of Medicine, The University of Sydney, Sydney, Australia \\ *Correspondence to nrod2590@uni.sydney.edu.au
}

Disclosure: $\quad$ The authors have declared no conflicts of interest.

Received: $\quad$ 17.07.19

Accepted: $\quad 02.01 .20$

Keywords: $\quad$ Biomarkers, gestational diabetes mellitus (GDM), macrosomia.

Citation:

EMJ. 2020;DOI/10.33590/emj/19-00150

\begin{abstract}
Gestational diabetes mellitus (GDM) is a frequent complication of pregnancy, with an increasing incidence that has been attributed to an ageing maternal population, an increasing prevalence of obesity, and alterations in diagnostic criteria. The consequences of GDM are far-reaching and impact both the mother and their offspring. It is associated with poor maternal and neonatal outcomes compared with non-GDM pregnancies. Furthermore, it is associated with long-term poor metabolic health in both mother and offspring. Current diagnostic strategies centre on clinical risk factors, however these can lack specificity. This has spurred investigations into identifying potential biomarkers to aid in diagnosis and risk stratification. In this review, the current evidence around potential biomarkers, their role in understanding pathophysiologic pathways for GDM development, and the possibility of their use in clinical practice is explored.
\end{abstract}

\section{INTRODUCTION}

Gestational diabetes mellitus (GDM), defined as glucose intolerance arising during pregnancy, is an increasingly prevalent condition, with consequences for perinatal and long-term health in both the mother and their offspring. Maternal complications include hypertensive disorders such as pre-eclampsia, necessity for assisted delivery including caesarean section, as well as an increased lifetime prevalence of Type 2 diabetes mellitus and cardiovascular disease. The offspring of women with GDM are also at increased risk of obesity and Type 2 diabetes mellitus in late adolescence and young adulthood, and they have higher rates of neonatal hypoglycaemia, jaundice, and polycythaemia, as well as macrosomia immediately after birth. ${ }^{1}$ Macrosomia, defined as birthweight $>4,000 \mathrm{~g}$, resulting from fetal overgrowth, independently increases the risk of birth complications such as shoulder dystocia, clavicle fracture, and brachial plexus injury, as well as predisposing to maternal complications such as caesarean delivery. ${ }^{2}$ Recent studies such as the Hyperglycaemia and Adverse Pregnancy Outcome Follow Up Study (HAPO FUS) have reiterated the association between high glucose exposure in utero, childhood insulin resistance, 
and metabolic dysregulation. ${ }^{3}$ Prevention, timely diagnosis, and early intervention are thus key to optimising not only maternal health, but also that of the offspring.

There is ongoing debate around what threshold serum glucose levels warrant a diagnosis of GDM. ${ }^{4}$ Objective, outcome-based measures would aid in optimising treatment in those most at risk of maternal and offspring adverse outcomes. The use of biomarkers for optimising accurate diagnosis and treatment of GDM is therefore of great interest to the clinical community and is currently the subject of intense research. This narrative review will describe the pathophysiology of GDM, and discuss potential biomarkers for GDM and macrosomia, highlighting the overlapping mechanisms of these conditions.

\section{PATHOPHYSIOLOGY OF GESTATIONAL DIABETES MELLITUS}

Insulin resistance (IR) and relative pancreatic $\beta$-cell dysfunction are central features of GDM. Normal physiology in pregnancy involves a gradual rise in $\mathrm{IR}$, such that target tissues, namely liver, adipose tissue, and muscle, have a decreased response to normal circulating insulin concentrations. ${ }^{5}$ This normal increase in IR allows the developing fetus to maintain an adequate supply of carbohydrates, while maternal energy supply is augmented by upregulated fat metabolism. ${ }^{6}$ The development of IR at a subclinical level appears to be key in the development of GDM. In fact, studies examining IR in early pregnancy demonstrate that women with higher IR in the first trimester are more likely to develop GDM at 28 weeks gestation. ${ }^{7}$ Higher serum insulin levels in early pregnancy are associated with increased risk of GDM at 28 weeks. ${ }^{8}$ The decline in insulin sensitivity is mediated by a reduction in the efficacy of insulin at the insulin receptor, attributed to rising concentrations of progesterone, cortisol, prolactin, and placental human lactogen. ${ }^{9}$ While these hormones are known to play an intrinsic role in the development of IR of pregnancy, changes in the concentrations of these hormones do not directly correlate with the rise in $I R .^{10}$ Other factors are at play to produce either exaggerated IR in pregnancy or relative insulin deficiency. Interestingly, ethnic variations in GDM prevalence point towards differences in $\beta$-cell capacity to overcome IR. For example, studies suggest that South Asian populations have a lower $\beta$-cell mass, contributing to a relative insulin deficiency underpinning their higher incidence of GDM. ${ }^{11}$ The role of $\beta$-cell mass in the development of insulin deficient states is further supported by studies in Type 2 diabetes mellitus, in which the transition period between normoglycaemia and Type 2 diabetes mellitus is characterised by a preceding decline in $\beta$-cell mass of $20-40 \% .^{12,13}$

\section{PATHOPHYSIOLOGY OF MACROSOMIA}

The major antecedent factor leading to macrosomia is maternal hyperglycaemia, most commonly seen in GDM. As maternal IR increases, relative maternal hyperglycaemia ensues and maternal glucose crosses the placenta to stimulate fetal insulin secretion, which in turn leads to increased fetal fat and protein stores with resultant macrosomia. ${ }^{2}$ In this context, the overgrowth pattern typically seen is one of asymmetric growth, with central deposition of subcutaneous fat around the abdomen and the back, greater shoulder circumference, and increased skin folds. ${ }^{14}$ The relationship between macrosomia and maternal glucose levels are wellestablished, with studies such as the Diabetes in Early Pregnancy Study linking neonatal birth weight with postprandial maternal blood glucose levels. ${ }^{15}$ Furthermore, data arising from the Australian Carbohydrate Intolerance Study in Pregnant Women (ACHOIS) study linked higher maternal fasting glucose levels in the third trimester of pregnancy with increased risk of shoulder dystocia. ${ }^{16}$ In a study examining diet controlled GDM, macrosomia rates were not increased compared to the control population, suggesting that normalisation of blood glucose levels reduces the risk of macrosomia. ${ }^{17}$

Independent of the development of maternal GDM, maternal obesity in early pregnancy is associated with macrosomia. ${ }^{18}$ Furthermore, women with excess gestational weight gain are more likely to have large for gestational age neonates with increased rates of caesarean delivery. ${ }^{19,20}$ The mechanisms proposed for these associations are likely multifactorial, with IR and hyperinsulinaemia being key contributing factors. ${ }^{21}$ Maternal hypertriglyceridaemia is increasingly recognised as important. ${ }^{22}$ In women without diabetes, second and third trimester triglyceride levels are positively correlated with birthweight, and is seen 
as an independent predictor of macrosomia. ${ }^{23}$ This association has also been illustrated in women with GDM, again independently of glycaemic control.24 Interestingly, restricting gestational weight gain targets in obese women with GDM does not improve perinatal outcomes, in particular macrosomia incidence, ${ }^{25}$ and lifestyle modification in early pregnancy does not reduce incidence rates of GDM. ${ }^{26}$ These findings suggest that while maternal triglyceride levels may be a potential biomarker for fetal macrosomia, other factors beyond maternal adiposity, IR, and GDM incidence are contributing to the development of macrosomia.

\section{INFLAMMATORY BIOMARKERS}

Markers of inflammation have been examined as possible biomarkers to predict GDM. In a prospective cohort study of nulliparous women, high leukocyte counts detected in early pregnancy were positively associated with the development of GDM, independent of known clinical risk factors for GDM. ${ }^{27}$ Leukocythemia may signify cytokine-induced IR, of which other inflammatory cytokines may also be involved in. Generally, high white cell counts are associated with increased adiposity, and predict worsening insulin action. In one study of Pima Indians, white blood cell mass showed a significant correlation with body fat percentage. ${ }^{28} \mathrm{IL}-6$ is secreted from adipocytes, with increased adipocyte mass associated with greater IL-6 levels. ${ }^{29}$ Interestingly, patients homozygous for IL-6 - 174 G/C, a polymorphism associated with reduced plasma concentration of IL- 6 , demonstrate lower levels of IR as measured through the glucose tolerance test. ${ }^{30}$ The association of IL- 6 with GDM is further strengthened by a study by Hassiakos et al.,31 who examined IL-6 levels at Weeks 11-14 of gestation. At this time in pregnancy, they found increased IL-6 concentrations in women who subsequently developed GDM, with a median of $0.5 \mathrm{pg} / \mathrm{mL}$ difference between GDM and non-GDM groups. ${ }^{31}$ IL-6 was the single serum predictor for GDM in this study. Combined with maternal characteristics, IL-6 yielded an improved prediction of GDM, with a detection rate of $67.5 \%$ compared with $59.0 \%$ on clinical factors alone, with a false positive rate of $25.0 \%{ }^{31} \mathrm{IL}-6$ levels were also positively correlated with GDM at the late second trimester, around the time of GDM diagnosis. ${ }^{32}$ Therefore,
IL-6 is a promising biomarker that may improve the prediction of GDM, even from early in the second trimester.

TNF-a is involved in normal IR of pregnancy and GDM pathogenesis. TNF-a is produced by both the placenta and adipose tissue, and its levels have been demonstrated to be higher in late pregnancy compared to early pregnancy, and significantly higher in women with GDM at the time of diagnosis compared with women without GDM. ${ }^{10}$ Its role in GDM may involve altered insulin sensitivity, through impairment of $\beta$-cell functioning and interference of insulin signalling pathways, as demonstrated through its inverse relationship with insulin sensitivity. ${ }^{10,33}$ Within in vitro cultures of placental and subcutaneous adipose tissue derived from women with GDM, TNF-a levels were significantly greater in response to high glucose exposure compared with women without GDM, suggesting that cells exposed to a GDM milieu are primed to be proinflammatory. ${ }^{34}$ Interestingly, in vitro modelling appears to favour the role of TNF-a in the maternal environment rather than the fetal environment, with greater levels expressed in maternal circulation compared with the fetal circulation. $^{10}$ Thus, while the association of TNF-a to GDM remains strong, its role as an independent biomarker for macrosomia is questionable.

C-reactive protein (CRP) is an acute-phase protein produced in response to tissue injury and inflammation. Type 2 diabetes mellitus and obesity are associated with increased low-grade, chronic inflammation, with CRP being a known independent risk factor for the development of the former. ${ }^{35,36}$ There is conflicting evidence about the relationship between CRP and GDM risk. In pregnancy, first trimester CRP levels correlate with increased GDM risk, with the highest levels of CRP being associated with a 3.5-fold increased risk of GDM compared to those with the lowest CRP levels. ${ }^{37}$ In this study, the relationship was independent of maternal weight, such that normal weight women had a 3.7-fold increased risk of GDM (95\% confidence interval: 1.6-8.7) if their serum CRP measurement was $>5.3 \mathrm{mg} / \mathrm{L}$ compared with $<5.3 \mathrm{mg} / \mathrm{L}^{37}$ Wolf et al. ${ }^{38}$ similarly found that a higher CRP level in the first trimester was significantly associated with subsequent GDM, only partly mediated by obesity. However, BMI was found to confound the association between CRP and GDM in a study by Berggren 
et al. ${ }^{39}$ The role of CRP in GDM appears, at least in part, to be interwoven with that of maternal obesity.

While inflammatory cytokines are emerging as important factors in the pathogenesis of GDM and macrosomia, they are not yet useful as biomarkers to incorporate into diagnostic tools for GDM prediction because of their lack of specificity. A global assessment of the inflammatory milieu may be helpful in the pregnancy to assess a potential risk for GDM, but at this stage current evidence does not yet distinguish a single inflammatory marker for use as a predictor of GDM or macrosomia. Proteomic analyses may prove to be useful in the analysis of the inflammasome and its predictive role in GDM.

\section{ADIPOKINES AS BIOMARKERS}

Adiposetissue is notastatic form ofenergy storage, but functions as an endocrine organ intrinsically linked to metabolism through the secretion of adipose-derived factors or adipokines. ${ }^{40}$ Leptin is one such adipokine, long-studied in relation to IR and obesity, involved in the regulation of feeding behaviour. ${ }^{41}$ Serum leptin levels are positively associated with adipocyte mass, demonstrating the development of leptin resistance, with attenuation of leptin signalling at a cellular level. ${ }^{41}$ In pregnancy, placental secretion of leptin further potentiates circulating concentrations, with levels peaking around the $28^{\text {th }}$ week of gestation, the time of physiological increase in IR. ${ }^{42}$ Women with GDM have significantly higher levels of circulating leptin compared with women without GDM, ${ }^{43}$ with higher leptin messenger RNA expression on the maternal tissue side of the placenta. Interestingly, women with pre-eclampsia, in which IR is also a feature, also have relative hyperleptinaemia. ${ }^{44}$

PPAR-y is a ligand-activated transcription factor predominantly involved in lipid metabolism, cell differentiation, and inflammatory responses. ${ }^{45}$ The role of PPAR-y in Type 2 diabetes mellitus is wellelucidated ${ }^{46}$ and exploited therapeutically using thiazolidinediones, in which activation of PPAR-Y leads to improved insulin sensitivity. The antiinflammatory action of this transcription factor has been studied in relation to Type 2 diabetes mellitus; PPAR-y upregulation in leukocytes leads to suppression of inflammatory cytokines such as TNF-a, IL-6, and IL-1B. ${ }^{47}$ PPAR-y expression in leukocytes isolated from the blood of pregnant women with GDM was positively associated with hyperglycaemia during glucose tolerance testing at 1 hour ( $r=0.222 ; p=0.049)$ and 2 hours ( $r=0.315$; $p=0.020$ ), suggesting that PPAR- $y$ expression is upregulated in women with GDM. ${ }^{48}$ To date, no studies have measured PPAR- $\mathrm{y}$ as a predictive marker for GDM in the first half of pregnancy.

Adiponectin, secreted from adipose tissue, is a known modulator of glucose metabolism, and is associated with greater insulin sensitivity and negatively associated with the development of Type 2 diabetes mellitus. ${ }^{49}$ In pregnancy, adiponectin levels decline with increasing gestation, ${ }^{50}$ exaggerated in women with GDM. ${ }^{51}$ Women with lower adiponectin levels in the first trimester have an increased risk of developing GDM in the second trimester; for each $1 \mu \mathrm{g} /$ $\mathrm{mL}$ decrease in adiponectin, there were 12\% increased odds of GDM development, adjusted for $\mathrm{BMI}$ and $\mathrm{HbA} 1 \mathrm{c}$ at first trimester. ${ }^{52}$ The converse relationship is seen with visfatin, for which the concentration is highest in the second trimester..$^{53}$ In a study examining pregnant women with preGDM and GDM, visfatin levels were significantly higher compared with women without GDM.54 Other adipokines have also been examined as possible biomarkers for GDM. SFRP- 5 is an anti-inflammatory adipokine downregulated in obesity. ${ }^{55}$ A small study of 40 women found that first trimester serum levels of SFRP- 5 were correlated with GDM development. Resistin, another adipokine known to increase during pregnancy and with increasing adiposity, has also been explored; however, overall no significant association has been demonstrated. ${ }^{56}$

While these biomarkers are interesting physiologically, their appeal as biomarkers for diagnostic purposes is hampered by their interconnected relationship with inflammation and obesity, thus reducing their specificity. Further studies are required to fully elucidate the role of adipokines in the pathogenesis of GDM and macrosomia. Furthermore, the metabolism of adipokines by the placenta needs further elucidation prior to implementation in clinical practice.

\section{BIOMARKERS OF PLACENTATION}

Biomarkers of placentation have been studied in 
relation to GDM and macrosomia, although the complex physiological interaction between the maternal-fetal-placental axis makes their use in GDM prediction difficult. Pregnancy associated plasma protein A (PAPP-A) and placental growth factor (PLGF) have shown variability in trends with respect to GDM, but overall have not been useful as first trimester screening tools for GDM. ${ }^{57}$ Macrosomia has been shown to be associated with increased PAPP-A, although its predictive value independent of maternal characteristics was not significant on linear regression. ${ }^{58} \mathrm{~A}$ study examining serum concentrations of various growth factors, including insulin like growth factor-1, and their receptor expression showed higher concentrations in women with GDM and, importantly, their corresponding macrosomic offspring, compared to controls. This is suggestive of a possible role of such growth factors in GDM and in macrosomia, through interaction at the maternal-fetal-placental axis. ${ }^{59}$ Sex hormone binding protein (SHBG) is a glycoprotein that regulates sex hormones. It is negatively associated with IR, with lower SHBG levels seen in women with GDM compared with matched controls at 24-28 weeks gestation. ${ }^{60}$ Furthermore, good predictive accuracy of SHBG was found for GDM requiring insulin therapy (area under the curve: 0.866; 95\% confidence interval: 0.773-0.959). ${ }^{61}$ Placental expression of SHBG has been examined in women with GDM compared to controls, with decreasing expression of SHBG protein and messenger RNA in women with GDM. ${ }^{62}$ This decrease in SHBG expression implies alteration of insulin signalling at the maternal-fetal interface, contributing to increased IR in these women. A greater understanding of the role of placental modulation by the maternal and fetal metabolic milieus is needed prior to incorporation of any such biomarkers into clinical practice.

\section{EMERGING TECHNIQUES}

High throughput technologies such as metabolomics offer potential insights into the pathophysiology of GDM. Metabolites associated with GDMincludeaminoacids, sphingomyelins, and glycerophospholipids, detected in the umbilical cord blood at the time of delivery. ${ }^{63}$ Proteins such as lactulose, uracil, and 2-methylfufurate were elevated in the cord blood of macrosomic neonates born to GDM compared with non-
GDM mothers. ${ }^{64}$ Interestingly, there seems to be very little overlap between the metabolomic profile in isolated macrosomia compared with isolated GDM, which is counterintuitive given the overlapping features of maternal adiposity, IR, and hypertriglyceridaemia. An interesting and currently unexplored line of enquiry would be to discern the role, if any, of fetal metabolism on GDM development. Furthermore, elucidation of the role of the placenta as a metabolic modulator of the maternal and fetal metabolome would provide potential novel insights into the pathogenesis of GDM and macrosomia.

Epigenetics is the study of chromosomal changes that do not affect the underling DNA sequence but regardless alter gene expression. DNA methylation is the most commonly examined epigenetic modification, involving covalent modification of the firth carbon of cytosine residues affecting gene transcription. In a study examining genome-wide methylation patterns of fetal cord blood from GDM pregnancies, significant methylation differences were identified at 65 CpG sites, ${ }^{65}$ and importantly were demonstrated to show association between maternal GDM and the epigenetic signatures of exposed offspring. Such findings allow greater exploration of gene candidates that may be responsible for transmitting the consequences of GDM, namely Type 2 diabetes mellitus and adolescent obesity, to the subsequent generation. With greater study, these may also prove useful biomarkers for diagnostic and prognostic clinical use. Currently, however, the complexities and costs attached to these techniques relegates them to research purposes only, at least presently.

\section{CONCLUSION}

The investigation into biomarkers associated with, and predictive of, GDM and macrosomia has provided insight into the pathophysiology, similarities, and differences of these conditions. So far, there have been no specific biomarkers that have clearly demonstrated potential for clinical utility, although many show great promise. Furthermore, the incorporation of insulin and lipid level screening in combination with clinical factors may enhance the identification of women at risk for babies with macrosomia. There is mounting evidence for markers of inflammation and adipokines as key regulators in the pathogenesis 
of GDM. With further exploration and validation, the integration of such biomarkers into diagnostic and therapeutic paradigms are likely to prove extremely useful in the prognostication of GDM and macrosomia. Furthermore, the use of novel investigative mechanisms such as metabolomics have the potential for further biomarker identification. Importantly, the cost-effectiveness of adding biomarker measurement to improve predictability of diagnosis and outcomes of GDM pregnancies requires evaluation prior to clinical application. While larger population studies are required to further validate such biomarkers prior to clinical use, their integration with clinical risk factors has the potential for greater stratification of perinatal and long-term metabolic risk for the pregnant woman and the unborn child, thereby minimising unnecessary therapy and targeting those women most at risk of adverse outcomes.

\section{References}

1. American Diabetes Association (ADA). Diabetes care. 2003;26(Suppl 1):s103-5.

2. $\mathrm{Kc} \mathrm{K}$ et al. Gestational diabetes mellitus and macrosomia: a literature review. Ann Nutr Metab. 2015;66(Suppl 2):14-20

3. Scholtens DM et al. Hyperglycemia and Adverse Pregnancy Outcome Follow-up Study (HAPO FUS): maternal glycemia and childhood glucose metabolism. Diabetes care. 2019;42(3):381-92.

4. Mclntyre HD et al. Gestational diabetes mellitus. Nat Rev Dis Primers. 2019;5(1):47.

5. Hunter SJ, Garvey WT. Insulin action and insulin resistance: diseases involving defects in insulin receptors, signal transduction, and the glucose transport effector system. Am J Med. 1998;105(4):331-45.

6. Sivan E et al. Effect of insulin on fat metabolism during and after normal pregnancy. Diabetes. 1999;48(4):8348.

7. Grewal E et al. Prediction of gestational diabetes mellitus at 24 to 28 weeks of gestation by using firsttrimester insulin sensitivity indices in Asian Indian subjects. Metabolism. 2012;61(5):715-20.

8. Bito $T$ et al. Prediction of gestational diabetes mellitus in a high-risk group by insulin measurement in early pregnancy. Diabetic Med. 2005;22(10):1434-9.

9. Ryan EA, Enns L. Role of gestational hormones in the induction of insulin resistance. J Clin Endocrinol Metab. 1988;67(2):341-7.

10. Kirwan JP et al. TNF-alpha is a predictor of insulin resistance in human pregnancy. Diabetes. 2002;51(7):2207-13.

11. Inaishi J, Saisho Y. Ethnic similarities and differences in the relationship between beta cell mass and diabetes. J Clin Med. 2017;6(12):113.

12. Butler AE et al. Beta-cell deficit and increased beta-cell apoptosis in humans with Type 2 diabetes. Diabetes. 2003;52(1):102-10.

13. Meier JJ et al. Pancreatic diabetes manifests when beta cell area declines by approximately $65 \%$ in humans. Diabetologia. 2012;55(5):1346-54.

14. McFarland MB et al. Anthropometric differences in macrosomic infants of diabetic and nondiabetic mothers. $J$ Matern-Fetal Med. 1998;7(6):292-5.

15. Jovanovic-Peterson $L$ et al. Maternal postprandial glucose levels and infant birth weight: the Diabetes in Early Pregnancy Study. Am J Obstet Gynecol. 1991;164(1 Pt 1):103-11.

16. Athukorala $\mathrm{C}$ et al. Women with gestational diabetes mellitus in the ACHOIS trial: risk factors for shoulder dystocia. Aust N Z J Obstet Gynaecol. 2007;47(1):37-41.

17. Vally $\mathrm{F}$ et al. Macrosomia rates in women with diet-controlled gestational diabetes: a retrospective study. J Pregnancy. 2017;2017:4935397.

18. Xiao L et al. Associations of maternal pre-pregnancy body mass index and gestational weight gain with birth outcomes in Shanghai, China. Sci Rep. 2017;7:41073.

19. Guelinckx I et al. Maternal obesity: pregnancy complications, gestational weight gain and nutrition. Obes Rev. 2008;9(2):140-50.

20. Sebire NJ et al. Maternal obesity and pregnancy outcome: a study of 287,213 pregnancies in London. Int J Obes Relat Metab Disord. 2001;25(8):1175-82.

21. Ahlsson $\mathrm{F}$ et al. Insulin resistance, a link between maternal overweight and fetal macrosomia in nondiabetic pregnancies. Horm Res Paediatr. 2010;74(4):267-74

22. Lindegaard $M L$ et al. Placental triglyceride accumulation in maternal type 1 diabetes is associated with increased lipase gene expression. J Lipid Res. 2006;47(11):2581-8.

23. Di Cianni $\mathrm{G}$ et al. Maternal triglyceride levels and newborn weight in pregnant women with normal glucose tolerance. Diabet Med. 2005;22(1):215.

24. Simeonova-Krstevska $S$ et al. Effect of lipid parameters on foetal growth in gestational diabetes mellitus pregnancies. Pril (Makedon Akad Nauk Umet Odd Med Nauki). 2014;35(2):131-6.

25. Wong $T$ et al. Are the Institute of Medicine weight gain targets applicable in women with gestational diabetes mellitus? Diabetologia. 2017;60(3):416-23.

26. Bain $\mathrm{E}$ et al. Diet and exercise interventions for preventing gestational diabetes mellitus. Cochrane Database Syst Rev. 2015(4):Cd010443.

27. Wolf $\mathrm{M}$ et al. Inflammation and glucose intolerance. Diabetes care. 2004;27(1):21.

28. Vozarova B et al. High white blood cell count is associated with a worsening of insulin sensitivity and predicts the development of Type 2 diabetes. Diabetes. 2002;51(2):455.

29. Bluher $M$ et al. Association of interleukin-6, C-reactive protein, interleukin-10 and adiponectin plasma concentrations with measures of obesity, insulin sensitivity and glucose metabolism. Exp Clin Endocrinol Diabetes. 2005;113(9):534-7.

30. Fernández-Real JM et al. Interleukin-6 gene polymorphism and insulin sensitivity. Diabetes. 2000;49(3):51720.

31. Hassiakos D et al. Increased maternal serum interleukin- 6 concentrations at 11 to 14 weeks of gestation in low risk pregnancies complicated with gestational diabetes mellitus: development of a prediction model. Horm Metab Res. 2016;48(1):35-41.

32. Siddiqui $\mathrm{S}$ et al. Association of IL-6 and CRP levels with gestational diabetes mellitus. Diabetes. 2018;67(Suppl 1):2417-PUB.

33. Cawthorn WP, Sethi JK. TNF-alpha and adipocyte biology. FEBS Lett. 
2008;582(1):117-31.

34. Coughlan MT et al. Glucose-induced release of tumour necrosis factoralpha from human placental and adipose tissues in gestational diabetes mellitus. Diabetic Med. 2001;18(11):921-7.

35. Ford ES. Body mass index, diabetes, and C-reactive protein among U.S. adults. Diabetes care. 1999;22(12):1971-7.

36. Pradhan $A D$ et al. C-reactive protein, interleukin 6 , and risk of developing Type 2 diabetes mellitus. JAMA. 2001;286(3):327-34.

37. Qiu C et al. A prospective study of maternal serum C-reactive protein (CRP) concentrations and risk of gestational diabetes mellitus. Paediatr Perinat Epidemiol. 2004;18(5):377-84.

38. Wolf $\mathrm{M}$ et al. First-trimester C-reactive protein and subsequent gestational diabetes. Diabetes Care. 2003;26(3):819-24.

39. Berggren EK et al. First-trimester maternal serum C-reactive protein as a predictor of third-trimester impaired glucose tolerance. Reprod Sci. 2015;22(1):90-3.

40. Hotamisligil GS. Inflammation and metabolic disorders. Nature. 2006;444(7121):860-7.

41. Friedman JM, Halaas JL. Leptin and the regulation of body weight in mammals. Nature. 1998;395(6704):763-70.

42. Briana D, Malamitsi-Puchner A Reviews: adipocytokines in normal and complicated pregnancies. Reprod Sci. 2009;16(10):921-37.

43. $\mathrm{Xu} \mathrm{J}$ et al. Maternal circulating concentrations of tumor necrosis factor-alpha, leptin, and adiponectin in gestational diabetes mellitus: a systematic review and metaanalysis. Scientific World Journal. 2014;2014:926932.

44. Laivuori $\mathrm{H}$ et al. Leptin during and after preeclamptic or normal pregnancy: its relation to serum insulin and insulin sensitivity. Metabolism. 2000;49(2):259-63.

45. Escher P, Wahli W. Peroxisome proliferator-activated receptors: insight into multiple cellular functions. Mutat Res. 2000;448(2):121-38.

46. He W et al. Adipose-specific peroxisome proliferator-activated receptor gamma knockout causes insulin resistance in fat and liver but not in muscle. Proc Natl Acad Sci USA. 2003;100(26):15712-7.

47. Jiang $\mathrm{C}$ et al. PPAR-gamma agonists inhibit production of monocyte inflammatory cytokines. Nature. 1998:391(6662):82-6.

48. Wójcik M et al. Gestational diabetes mellitus is associated with increased leukocyte peroxisome proliferatoractivated receptor $\mathrm{y}$ expression. Arch Med Sci. 2015;11(4):779-87.

49. Tabák AG et al. Adiponectin trajectories before Type 2 diabetes diagnosis: Whitehall II study. Diabetes Care. 2012;35(12):2540-7.

50. Galic S et al. Adipose tissue as an endocrine organ. Mol Cell Endocrinol. 2010:316:129-39.

51. Doruk $M$ et al. Serum adiponectin in gestational diabetes and its relation to pregnancy outcome. J Obstet Gynaecol. 2014;34(6):471-5.

52. Lacroix $M$ et al. Lower adiponectin levels at first trimester of pregnancy are associated with increased insulin resistance and higher risk of developing gestational diabetes mellitus. Diabetes Care. 2013;36(6):1577-83.

53. Mazaki-Tovi S et al. Maternal visfatin concentration in normal pregnancy. J Perinat Med. 2009;37:206-17.

54. Coskun A et al. Plasma visfatin levels in pregnant women with normal glucose tolerance, gestational diabetes and pre-gestational diabetes mellitus. J Matern Fetal Neonatal Med. 2010;23(9):1014-8.

55. Ouchi $\mathrm{N}$ et al. Sfrp5 is an anti-inflammatory adipokine that modulates metabolic dysfunction in obesity. Science. 2010;329(5990):454-7.

56. Lobo $\mathrm{T}$ et al. Resistin concentration and gestational diabetes: a systematic review of the literature. $J$ Reprod Immunol. 2013;97:120-7.

57. Syngelaki A et al. First-trimester biochemical markers of placentation in screening for gestational diabetes mellitus. Metabolism. 2015:64(11):1485-9.

58. Poon LC et al. First-trimester prediction of macrosomia. Fetal Diagn Ther. 2011;29(2):139-47.

59. Grissa $\mathrm{O}$ et al. Growth factor concentrations and their placental mRNA expression are modulated in gestational diabetes mellitus: possible interactions with macrosomia. BMC Pregnancy and Childbirth. 2010;10:7.

60. Bartha J et al. Sex hormone-binding globulin in gestational diabetes. Acta Obstet Gynecol Scand. 2000;79(10):839-45.

61. Caglar $\mathrm{G}$ et al. Sex-hormone-binding globulin early in pregnancy for the prediction of severe gestational diabetes mellitus and related complications. J Obstet Gynaecol Res. 2012;38(11):1286-93.

62. Zhang $B$ et al. Expression and correlation of sex hormonebinding globulin and insulin signal transduction and glucose transporter proteins in gestational diabetes mellitus placental tissue. Diabetes Res Clin Pract. 2016;119:106-17.

63. Lu YP et al. Fetal serum metabolites are independently associated with gestational diabetes mellitus. Cell Physiol Biochem. 2018;45(2):625-38

64. Sun $\mathrm{H}$ et al. Metabolic profiling of umbilical cord blood in macrosomia. Int J Obes (Lond). 2018;42(4):679-85

65. Haertle L et al. Epigenetic signatures of gestational diabetes mellitus on cord blood methylation. Clin Epigenetics. 2017;9:28 